\title{
The effects of pre-transplantation center-based cardiac rehabilitation on the postoperative quality of life and adherence to exercise in patients undergoing heart transplantation
}

\author{
Yong-Gon Seo ${ }^{1}$, Won-Hah Park ${ }^{1}$, Suki Oh ${ }^{1}$, Eun-Seok Jeon ${ }^{2}$, Jin-Oh Choi ${ }^{2}$, Ho-Young Kim ${ }^{3}$, \\ Mija Jang ${ }^{4}$, Yang-Hyun Cho ${ }^{5}$, Jidong Sung ${ }^{2, *}$ \\ ${ }^{1}$ Division of Sports Medicine, Department of Orthopedic Surgery, Samsung Medical Center, 06351 Seoul, Republic of Korea \\ ${ }^{2}$ Division of Cardiology, Department of Internal Medicine, Samsung Medical Center, 06351 Seoul, Republic of Korea \\ ${ }^{3}$ Cardiac Rehabilitation and Prevention Center, Samsung Medical Center, 06351 Seoul, Republic of Korea \\ ${ }^{4}$ Department of Nursing, Cardiac Rehabilitation and Prevention Center, Samsung Medical Center, 06351 Seoul, Republic of Korea \\ ${ }^{5}$ Department of Thoracic and Cardiovascular Surgery, Samsung Medical Center, 06351 Seoul, Republic of Korea \\ *Correspondence: jidong.sung@samsung.com (Jidong Sung) \\ Academic Editors: Kazuhiro P. Izawa, Peter H. Brubaker and Giuseppe Santarpino \\ Submitted: 19 August 2021 Revised: 9 October 2021 Accepted: 20 October 2021 Published: 17 February 2022
}

\begin{abstract}
Background: Heart transplantation (HTx) is the final treatment option for patients with chronic heart failure. Several studies have reported that exercise therapy, which is a component of cardiac rehabilitation, improves exercise capacity and the quality of life (QOL) in patients with heart failure. Methods: Three patients, referred to an inpatient cardiac rehabilitation before HTx, participated in centerbased cardiac rehabilitation (CBCR) during the hospitalization period. The CBCR, including aerobic exercises and resistance training, was conducted 3-4 days/week under cardiac rehabilitation team. Clinical outcomes included QOL using short form (SF)-36 questionnaire and the level of physical activity using international physical activity questionnaire (IPAQ) were evaluated before and after following the CBCR. Results: These patients showed an improved QOL in physical components with mean $32 \%$ and in mental components with mean $43 \%$. The adherence to exercise confirmed by level of physical activity also increased as much as mean 1275 MET-minutes at 3 months after transplantation. Conclusions: The findings in case report show the safety and effectiveness of CBCR in patients waiting for HTx during hopitalization. This is the first case report showing the effect of CBCR in admission period in Korean patients undergoing HTx.
\end{abstract}

Keywords: cardiac rehabilitation; heart transplantation; quality of life; patient compliance

\section{Introduction}

The prevalence of heart failure (HF) in Korea was estimated to be $1.53 \%$ in 2013 and is expected to increase to $3.35 \%$ by 2040 as a consequence of aging of the population [1]. Heart transplantation (HTx) is a treatment option for patients with advanced HF, and 1319 patients have undergone HTx between 1992 and 2016 in Korea [2].

Exercise-based cardiac rehabilitation is a safe intervention that improves short-term exercise capacity in heart transplant recipients [3]. Participation in cardiac rehabilitation following HTx has been reported to be relatively low, ranging from $43.3 \%$ to $55 \%$ [4,5]. Most previous studies have reported the effect of exercise-based cardiac rehabilitation after HTx on exercise capacity, the quality of life (QOL), and readmission [3-5].

Several studies [6-8] have suggested that preoperative exercise therapy including aerobic exercise, inspiratory muscle training, balance exercise, and strength exercise is beneficial in reducing postoperative complications and the length of hospital stay in patients undergoing cardiac surgery. As the average waiting time for HTx has increased over the past years, psychological, behavioral, and social problems have also increased among patients waiting for or undergoing HTx, and these problems are associated with post-transplantation complications [9]. However, there is a lack of evidence showing that pre-transplantation exercise therapy improves exercise capacity, the quality of life, and adherence to exercise after transplantation.

Therefore, the purpose of this case report was to identify the effect of pre-transplantation center-based cardiac rehabilitation (CBCR) during hospitalization on QOL and adherence to exercise at 3 months post-transplantation in patients undergoing HTx.

\section{Details of clinical cases}

The first patient was a 35-year-old woman who was diagnosed with dilated cardiomyopathy (DCMP). She underwent cardioverter-defibrillator implantation following a cardiac arrest in 2013. Despite optimal medical treatment and interventional therapy, severe dyspnea persisted. Therefore, the patient was admitted to the S Medical Center on March 5, 2018, and underwent HTx on October 31, 2018. She waited for 241 days in the hospital for HTx. The patient began to participate in inpatient cardiac rehabilita- 
A

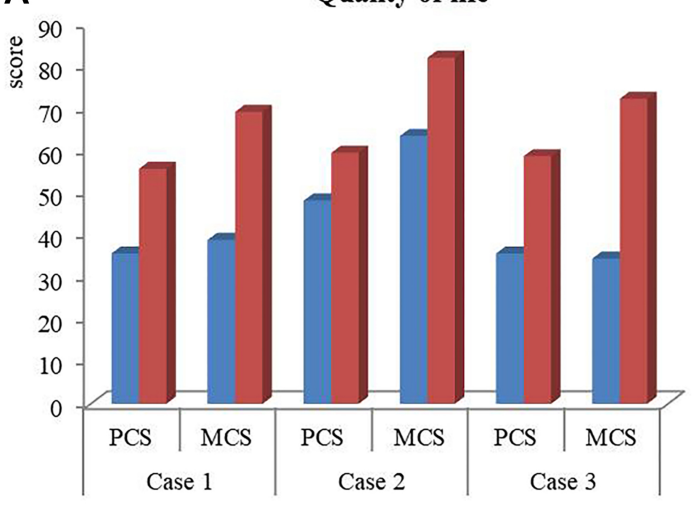

B

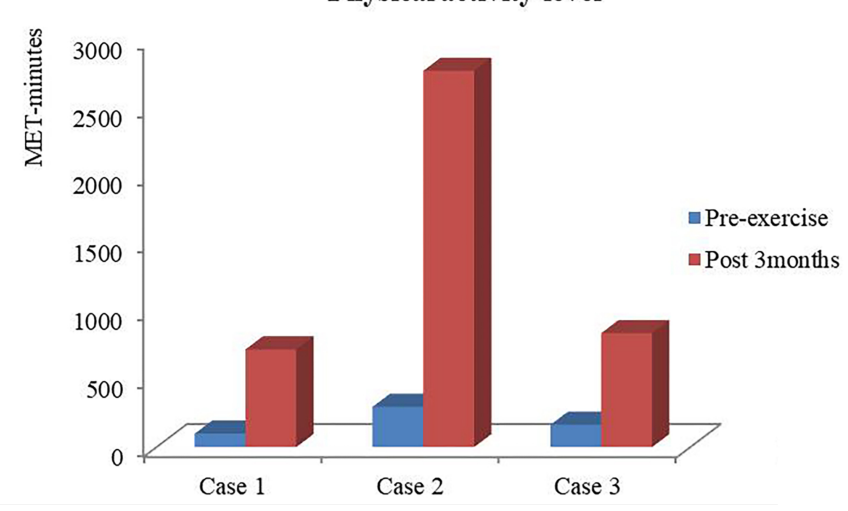

Fig. 1. Clinical outcomes after center-based cardiac rehabilitation. (A) The SF-36 questionnaire was used to evaluate the quality of life. A higher score indicates better quality of life. (B) Adherence to exercise was confirmed using the International Physical Activity questionnaire. Higher scores indicate higher physical activity levels. PCS, physical component score; MCS, mental component score.

Table 1. Characteristic of patients and clinical outcomes.

\begin{tabular}{lccc}
\hline Variables & Case 1 & Case 2 & Case 3 \\
\hline Sex & Female & Female & Female \\
Age (year) & 35 & 59 & 26 \\
Blood type & $\mathrm{O}$ & $\mathrm{A}$ & $\mathrm{O}$ \\
Height $(\mathrm{cm})$ & 166 & 152 & 163 \\
Weight $(\mathrm{kg})$ & 73.3 & 53.4 & 70.5 \\
Body mass index $\left(\mathrm{m}^{2} / \mathrm{kg}\right)$ & 26.6 & 23.1 & 26.5 \\
Diagnosis & DCMP & DCMP & DCMP \\
Ejection fraction $(\%)$ & 30.3 & 25.1 & 20.3 \\
VO ${ }_{2}$ peak (ml $\left./ \mathrm{kg} / \mathrm{min}\right)$ & 15.7 & 7.8 & 9.9 \\
Medical history & Atrial fibrillation & CTx induced CMP & Atrial flutter \\
& Hypothyroidism & Anomalous origin of the coronary & WPW syndrome \\
& Cardiac arrest & artery (RCA from LCC) & r/o TIA \\
HTx waiting period (day) & s/p ICD insertion & Breast cancer op & Hypothyroidism \\
Hospitalization after HTx (day) & 241 & 142 & 77 \\
Planned readmission (day) & 21 & 26 & 20 \\
Unplanned readmission (day) & 5 & 3 & 2 \\
Adverse event in CBCR & 0 & 0 & 0 \\
\hline
\end{tabular}

DCMP, dilated cardiomyopathy; HTx, heart transplantation; ICD, implantable cardioverter defibrillator; CTx, chemotherapy; RCA, right coronary artery; $\mathrm{CBCR}$, center-based cardiac rehabilitation; LCC, left coronary cusp; WPW, Wolff-Parkinson-White; TIA, transient ischemic attack; $\mathrm{VO}_{2}$, oxygen uptake; CMP, cardiomyopathy; r/o, rule out; s/p, status post; op, operation.

tion at the S Medical Center on April 20, 2018. The second patient was a 59-year-old woman who was diagnosed with DCMP induced by chemotherapy. She had an anomalous origin of the right coronary artery and had undergone mastectomy for breast cancer on May 30, 2007. The patient received medical treatment with medication and intervention, but her dyspnea worsened while performing activities of daily living. HTx was planned to improve severe dyspnea and the QOL, and the patient was admitted to our hospital on September 20, 2018. After waiting for 142 days, the patient underwent HTx on February 8, 2019. The patient also participated in center-based pre-transplantation cardiac rehabilitation from November 12, 2018 to before the day of HTx. The third patient was a 26-year-old woman with atrial flutter and Wolff-Parkinson-White syndrome who had been diagnosed with DCMP with left ventricular systolic dysfunction. On echocardiography, the ejection fraction was $20.3 \%$, and the wall motion of the heart was hypoactive. Her shortness of breath worsened, and she could not maintain normal activity due to systemic fatigue. Therefore, the 
Table 2. The center-based cardiac rehabilitation program.

\begin{tabular}{|c|c|c|c|}
\hline Variables & Case 1 & Case 2 & Case 3 \\
\hline Exercise frequency & 4-5/week & $3-4 /$ week & $3-4 /$ week \\
\hline Aerobic exercise intensity & Resting HR $+20-30$ & Resting HR $+20-30$ & Resting HR $+20-30$ \\
\hline (initial/final) & $\mathrm{bpm} / \mathrm{RPE} 12-13$ & $\mathrm{bpm} / \mathrm{RPE} 12-13$ & $\mathrm{bpm} / \mathrm{RPE} 12-13$ \\
\hline Bike: initial workload & Level $0,8 \mathrm{~min}$ & Level $0,10 \mathrm{~min}$ & Level $0,10 \mathrm{~min}$ \\
\hline Final workload & Level 0,15 min & Level $0-1,15 \mathrm{~min}$ & Level 0,15 min \\
\hline Treadmill: initial workload & $\mathrm{S} 4.0, \mathrm{G} 010 \mathrm{~min}$ & $\mathrm{~S} 3.4$, G $010 \mathrm{~min}$ & $\mathrm{~S} 3.2$, G $010 \mathrm{~min}$ \\
\hline Final workload & $\mathrm{S} 4.5, \mathrm{G} 115 \mathrm{~min}$ & $\mathrm{~S} 4.2, \mathrm{G} 115 \mathrm{~min}$ & $\mathrm{~S} 4.2$, G $015 \mathrm{~min}$ \\
\hline RT: initial workload & ${ }^{a}$ Mat exercise, RPE $11-13$ & Mat exercise, RPE 11-13 & Mat exercise, RPE 11-13 \\
\hline \multirow{2}{*}{ Final workload } & ${ }^{b}$ Machine exercise, $10-15$ & Machine exercise, $10-15$ & Machine exercise, $10-15$ \\
\hline & Rep/2-3 sets & Rep/2-3 sets & Rep/2-3 sets \\
\hline Total exercise time (min) & 60 & 60 & 60 \\
\hline Exercise period (month) & 6 & 3 & 2 \\
\hline Total sessions of CBCR (day) & 103 & 33 & 30 \\
\hline Frequency of RT (day) & 77 & 27 & 16 \\
\hline Exercise procedure & \multicolumn{3}{|c|}{$\begin{array}{l}\text { Stretching for warm-up (5-10 min), aerobic exercise using a stationary bike and treadmill } \\
(25-30 \mathrm{~min}) \text {, mat or weight training exercise }(15-20 \mathrm{~min}) \text {, stretching for cool-down }(5-10 \mathrm{~min})\end{array}$} \\
\hline
\end{tabular}

patient was admitted to the S Medical Center on January 17, 2019, and waited for 77 days until HTx. She started inpatient cardiac rehabilitation at the S Medical Center on January 20, 2019, and participated until HTx (Table 1). Patient consent was obtained from all subjects involved in the study.

CBCR was conducted for these patients after hospitalization at the S Medical Center, according to the recommendation of a cardiac specialist. The first exercise was stretching for warm-up, followed by aerobic exercises using a bicycle and treadmill machine. The bicycle exercise was for initial aerobic exercise and started at level 0 for 5 or 8 min according to each patient's exercise capacity or condition. The target heart rate for aerobic exercise was calculated as the resting heart rate plus 20 beats per minute or perceived exertion using the Borg scale of perceived exertion (range, 11-13). Resistance training was started after 1 month of aerobic exercise if the patient did not develop symptoms or other cardiac problems. The initial resistance training used the patient's body weight; later, the intensity was increased using weight training equipment (Table 2). The electrocardiogram was checked using a Q-Tel telemetry device (Quinton Instrument Co, Bothell, WA, USA) to monitor patient status.

To evaluate the QOL, the Short-Form 36 Questionnaire (SF-36), including a physical component and mental component, was administered in before beginning rehabilitation and then postoperative 3 months. The SF- 36 scores of all participants increased in postoperative 3 months compared to before CBCR. The physical component scores in- creased in all participants: pre-CBCR versus post-CBCR scores in cases 1, 2, and 3 were 35.6 versus 55.6, 48.1 versus 59.4, and 35.6 versus 58.6, respectively. Similarly, the mental component scores of all patients also improved: preCBCR versus post-CBCR scores in cases 1,2 , and 3 were 38.8 versus $69.1,63.4$ versus 81.9 , and 34.4 versus 72.2 , respectively. The International Physical Activity Questionnaire (IPAQ; Korean version) [10] also indicated improvement in physical activity after postoperative 3 months in all patients. Physical activity of patients 1,2, and 3 increased from 99 metabolic equivalents (MET)-minutes, 297 METminutes, and 165 MET-minutes to 720 MET-minutes, 2772 MET-minutes, and 840 MET-minutes, respectively (Fig. 1).

\section{Discussion}

CBCR is usually recommended after discharge from the hospital to facilitate early recovery and prevent other cardiac illnesses. In general, inpatient cardiac rehabilitation for patients waiting for HTx is conducted in the ward and includes bed exercise and corridor walking [11]. In this report, CBCR was conducted at the S Medical Sports Center during hospitalization, and patients showed improvement in the QOL and adherence to exercise confirmed by using IPAQ after HTx. Preoperative exercise before cardiac surgery is a beneficial intervention to improve postoperative clinical outcomes [6-8]. Unlike study subjects in previous studies, this case report was to examine the effects of CBCR before transplantation in cardiac patients who were in hospitalization, and we confirmed its feasibility and safety. 
HTx candidates have psychosocial problems, such as depression and social isolation, which seem to negatively affect health behavior and compliance [8]. In this report, the SF-36 questionnaire was used to evaluate the QOL at 3 months postoperatively and indicated improvement in physical (mean 32\%) and mental health (mean 42\%). Mental health improved more than physical health. Regular contact with the cardiac rehabilitation team during CBCR may have been an important factor in improving the mental health aspects of the QOL. Further controlled studies with a high number of patients are needed to identify the effectiveness of pre-transplantation CBCR on the QOL after posttransplantation.

Participation in exercise after HTx is advised to improve exercise capacity and increase physical activity. Home-based exercise is usually recommended because it can achieve similar improvements in postoperative clinical outcomes compared to $\mathrm{CBCR}[6,12,13]$. In this report, we measured adherence to exercise using a self-reported IPAQ to calculate the score of physical activity at 3 months postoperatively. All patients maintained physical activity after discharge from the hospital to 3 months posttransplantation, and the exercise volume was also increased after HTx. All patients have enrolled and attended in outpatient cardiac rehabilitation after postoperative 3 months. Adherence to exercise can be assessed using subjective or objective methods. We used a subjective method in the form of a physical activity questionnaire. Therefore, further studies are needed to measure adherence to exercise using pedometers or accelerometers to obtain detailed information.

This report had some limitations. This was a small case series of only three patients. Therefore, the results are difficult to generalize to all patients waiting for HTx. Second, this report did not include a control group for comparison. Therefore, the results in this study may be considered as a report showing the feasibility and safety of CBCR before HTx.

\section{Conclusions}

This case report showed pre-transplantation CBCR during hospitalization may be helpful to improve QOL and adherence to exercise at 3 months postoperatively after heart transplantation. The findings show the feasibility and safety of CBCR in patients waiting for HTx in admission period, and further study with large subjects is required to confirm this result by comparing with control group.

\section{Author contributions}

YGS, WHP, and JS designed the research study. YGS, SO, HYK, and MJ performed the research. ESJ, JOC, and YHC provided help and assist the research. YGS, SO, HYK, and MJ corrected and analyzed the data. YGS wrote the manuscript with support from JS, ESJ, JOC, WHP and YHC. All authors read and approved the final manuscript.

\section{Ethics approval and consent to participate}

Patient consent was obtained from all subjects involved in the study.

\section{Acknowledgment}

We thank the professors who refer cardiac patients to our cardiac rehabilitation team in Samsung Medical Center.

\section{Funding}

This research received no external funding.

\section{Conflict of interest}

The authors declare no conflict of interest.

\section{References}

[1] Lee JH, Lim NK, Cho M, Park HY. Epidemiology of Heart Failure in Korea: Present and Future. Korean Circulation Journal. 2016; 46: 658-664.

[2] Lee HY, Oh BH. Heart Transplantation in Asia. Circulation Journal. 2017; 81: 617-621.

[3] Anderson L, Nguyen TT, Dall CH, Burgess L, Bridges C, Taylor RS. Exercise-based cardiac rehabilitation in heart transplant recipients. The Cochrane Database of Systematic Reviews. 2017; 4: CD012264.

[4] Bachmann JM, Shah AS, Duncan MS, Greevy RA, Graves AJ, $\mathrm{Ni} \mathrm{S}$, et al. Cardiac rehabilitation and readmissions after heart transplantation. The Journal of Heart and Lung Transplantation. 2018; 37: 467-476.

[5] Bachmann JM, Shah A, Graves AJ, Ni S, Wang T, Whooley M, et al. Cardiac rehabilitation and readmissions after heart transplantation. The Journal of the American College of Cardiology. 2016; 67: 1856 .

[6] Waite I, Deshpande R, Baghai M, Massey T, Wendler O, Greenwood S. Home-based preoperative rehabilitation (prehab) to improve physical function and reduce hospital length of stay for frail patients undergoing coronary artery bypass graft and valve surgery. The Journal of Cardiothoracic Surgery. 2017; 12: 91.

[7] Drudi LM, Tat J, Ades M, Mata J, Landry T, MacKenzie KS, et al. Preoperative Exercise Rehabilitation in Cardiac and Vascular Interventions. The Journal of Surgical Research. 2019; 237: 311.

[8] Hulzebos EHJ, Smit Y, Helders PPJM, Meeteren NLU. Preoperative physical therapy for elective cardiac surgery patients. The Cochrane Database of Systematic Reviews. 2012; 11: CD010118.

[9] Spaderna H, Smits JMA, Rahmel AO, Weidner G. Psychosocial and behavioural factors in heart transplant candidates-an overview. Transplant International. 2007; 20: 909-920.

[10] Oh JY, Yang YJ, Kim BS, Kang JH. Validity and reliability of Korean version of International Physical Activity Questionnaire (IPAQ) short form. Journal of the Korean Academy of Family Medicine 2007; 28: 532-541.

[11] Seo YG, Jang MJ, Park WH, Hong KP, Sung J. Inpatient cardiac rehabilitation programs' exercise therapy for patients undergoing cardiac surgery: National Korean Questionnaire Survey. The Journal of Exercise Rehabilitation. 2017; 13: 76-83.

[12] Anderson L, Sharp GA, Norton RJ, Dalal H, Dean SG, Jolly K, et al. Home-based versus centre-based cardiac rehabilitation. The Cochrane Database of Systematic Reviews. 2017; 6: CD007130.

[13] Thomas RJ, Beatty AL, Beckie TM, Brewer LC, Brown TM, Forman DE, et al. Home-Based Cardiac Rehabilitation: A Scientitic From the American Association of Cardiovascular and Pulmonary Rehabilitation, the American Heart Association, and the American College of Cardiology. Journal of the American College of Cardiology. 2019; 74: 133-153. 